\title{
Recent advances in biomarkers in severe burns
}

Mireia Ruiz-Castilla ${ }^{1,2}$, Oriol Roca ${ }^{3,4}$, Joan R Masclans ${ }^{4,5}$, Joan P Barret ${ }^{1,2}$

${ }^{1}$ Plastic and Reconstructive Surgery Department and Burns Unit, Valld'Hebron University Hospital, Valld'Hebron Research Institute, Barcelona, SPAIN.

${ }^{2}$ Surgery Department, UniversitatAutònoma de Barcelona, Bellaterra, SPAIN.

${ }^{3}$ Critical Care Department, Valld'Hebron University Hospital, Valld'Hebron Research Institute, Barcelona, SPAIN.

${ }^{4}$ Ciber Enfermedades Respiratorias (Ciberes), Instituto de Salud Carlos III, Madrid, SPAIN.

${ }^{5}$ Critical CareDepartment, Parc de Salut Mar (Hospital del Mar) de Barcelona, IMIM (Hospital del Mar Medical Research Institute), Barcelona, SPAIN.

Corresponding author:

Mireia Ruiz Castilla MD

Plastic Reconstructive Surgery Department and Burns Unit

Valld'Hebron University Hospital

Pg Valld'Hebron 119-129

08035 Barcelona, SPAIN

Phone: +34

e-mail: miruiz@vhebron.net

The authors have no conflicts of interest to disclose. 


\section{ABSTRACT}

The pathophysiology of burn injuries is tremendously complex. A thorough understanding is essential for correct treatment of the burned area and also to limit the appearance of organ dysfunction, which, in fact, is a key determinant of morbidity and mortality. In this context, research into biomarkers may play a major role. Biomarkers have traditionally been considered as an important area of medical research: the measurement of certain biomarkers has led to a better understanding of pathophysiology, while others have been used either to assess the effectiveness of specific treatments or for prognostic purposes. Research into biomarkers may help to

improve the prognosis of patients with severe burn injury. The aim of the present clinical review is to discuss new evidence of the value of biomarkers in this setting. 


\section{INTRODUCTION}

Burn injury is a common type of traumatic injury. A recent European study estimated the annual incidence of severe burns at between 0.2 and 2.9/10,000 inhabitants ${ }^{1}$. Burns cause considerable morbidity and mortality, accounting for over 300,000 deaths per year worldwide ${ }^{2}$, and affect long-term health-related quality of life ${ }^{3}$. Burns are also among the most expensive traumatic injuries, due to the need for long-term hospitalization and rehabilitation periods and costly wound and scar treatment ${ }^{4,5}$.

Different definitions of biomarkers have been proposed. They could be defined as characteristics that can be objectively measured and evaluated as an indicator of normal biological processes, pathogenic processes, or pharmacologic responses to a therapeutic intervention ${ }^{4}$. In this regard, the WHO proposed a broad definition of biomarker including "almost any measurement reflecting an interaction between a biological system and a potential hazard, which may be chemical, physical, or biological. The measured response may be functional and physiological, biochemical at the cellular level, or a molecular interaction" ${ }^{5}$.Therefore, a biomarker can be considered as everything from the pulse, blood pressure or urine output through basic molecules present in blood and tissues.

The ideal biomarker should be easily and rapidly obtained and must be sensitive, specific and reproducible. Its levels should be modified by therapeutic interventions and it should also be useful for prognostic purposes. Ideally, the processes of procurement and measurement must also be cost-effective. Research into biomarkers has become increasingly important for several reasons. These indicators have helped us to understand the pathophysiology of diseases, allowing early and accurate diagnosis of particular complications and determining the severity of illness. Moreover, they may also be useful for designing new therapeutic targets that can improve outcomes, and for assessing the effectiveness of treatments. Therefore, the aim of this 
review is to discuss the recent advances in research into the utility of biomarkers in burn injury. 


\section{UNDERSTANDING THE PATHOPHYSIOLOGY OF BURN INJURY}

\section{The importance of inflammation in burn injury}

Understanding the pathophysiology of burn injury is crucial for correct treatment. Burn injuries trigger both local and systemic responses. Indeed, excessive burn load may cause a disproportionate immune response and patients may develop exaggerated systemic inflammatory processes. Thus, the question of how the multiorgan failure occurs in severe burned patient has aroused particular interest. Patients with severe burns are likely to suffer from sepsis that can rapidly develop into a systemic inflammatory response syndrome, which may simultaneously damage many organs such as the lung, the liver, the kidney, the gut or the brain.

In this regard, some studies have investigated the different inflammatory mediators concentration after burn injury. Interleukin (IL)1 receptor antagonist (IL1Ra) has been shown to be increased from the day of admission in patients with second and third degree thermal injuries ${ }^{6}$ and its concentrations were significantly higher in nonsurvivors with inhalation injury (Table 1). IL1Ra has been also correlated with total body surface area (TBSA) during the first week following burn injury ${ }^{7}$. Interestingly, IL1Ra levels were significantly higher in patients with severe sepsis, septic shock and multiorgan dysfunction syndrome (MODS). IL1Ra concentration correlated with other inflammatory mediators, such as IL6, IL8 and tumor necrosis factor (TNF) $\alpha$, and patient's clinical status regardless the presence of infection. Moreover, IL1Ra levels at admission and its maximum levels were significantly higher in those patients who finally died. Vindenes et $\mathrm{al}^{8}$ reported significant correlation between IL1Ra concentration and TBSA and the area of third degree burn, as well as with plasma concentrations of $\mathrm{C}$ - reactive protein (CRP). Furthermore, higher concentrations of IL1 $\beta$ and IL1ra were observed in patients who developed infective complications and patients who survived had significantly higher IL1 $\beta$ concentrations. Other studies have also shown that IL6, IL8 and TNFa concentrations are increased in serum after severe injury and they were 
significantly higher in the septic patients, who all died ${ }^{9-11}$. Moreover, IL10 concentration has been shown to be higher in nonsurvivors burn patients with proven sepsis ${ }^{12}$. In contrast, a lower second peak of transforming growth factor $\beta 1$ (TGF $\beta 1$ ) has been observed in septic nonsurvivors burn patients ${ }^{13}$. More recently, it has been shown that several serum cytokines measured are significantly altered after severe burn injury in a large cohort of pediatric burn patients. A marked increase in granulocyte colonystimulating factor (G-CSF), IL6, IL8, monocyte chemoattractant protein-1 (MCP1), and macrophage inflammatory protein-1 $\beta$ (MIP1 $\beta)$ has been observed when compared to normal levels ${ }^{14}$. This hyperinflammation may compromise multiple organ functions such as the lung, kidney, liver, bowel and brain ${ }^{15}$. In fact, severe burns may alter the function of physiological barriers, allowing inflammatory cells to pass and release amounts of cytokines, reactive oxygen species (ROS), complement proteins and other agents that aggravate the organ damage ${ }^{16}$. In this regard, in a large cohort of patients with severe burn injury, defined as more than $30 \%$ of TBSA, higher concentrations of IL6, MCP1, $\operatorname{TNF} \alpha$, and CRP have been observed in patients with multiorgan failure compared with those patients without ${ }^{17}$. Equally, another recent study that included 230 pediatric burns with $>30 \%$ of TBSA reported higher serum levels of IL6, IL8, G-CSF, MCP1, C-reactive protein in nonsurvivors ${ }^{18}$. All these results suggest that inflammatory reaction plays an important role in burn injury pathophysiology and it may also be important in organ dysfunction generation as well as it may also predict its outcomes.

Significantly, multiorgan dysfunction syndrome (MODS) has been reported as a main cause of death in burn patients ${ }^{19}$; in fact, in a large cohort of burn patients, MODS was almost invariably present in the patients who died ${ }^{20}$. Consequently, in the overall treatment of burned patients, just as important as the surgical management of the burn are the general supportive measures to minimize the occurrence of organ failure. Therefore, gaining an understanding of the exact mechanisms involved in burn injury and the genesis of the associated organ dysfunction will help to develop novel 
therapeutic strategies which can allow successful modulation of the inflammation and improve outcomes. In this regard, despite the inflammatory response, the host's response to burn injury also plays an important role. It has recently been shown that an overwhelming anti-inflammatory response may be associated with worse prognosis. In this sense a cut off of $14 \mathrm{pg} / \mathrm{ml}$ of IL10 has demonstrated a good discrimination capability between survivors and those burn patients who will not survive ${ }^{21}$. On the other hand, several CD markers of granulocytes, lymphocytes and monocytes have been shown to be overexpressed in survivors ${ }^{22}$.

\section{Fungal wound infection}

Historically, infection has been described as one of the main causes of morbidity and mortality in these patients ${ }^{23}$. In a study that included more than 2500 burn patients, fungal wound infection was independently associated with mortality regardless of age, burn size or the presence of inhalation injury ${ }^{24}$. Although bacterial infections are probably the most common infections in burn patients, severe burn patients also present a higher risk of fungal infection. Some studies have tried to explain this issue. Inatsu et al ${ }^{25}$, stimulated peripheral blood mononuclear cells (PBMCs) of healthy volunteers and burn patients with Candida albicans antigen (CAg). Burn patient sera were shown to be inhibitory on CAg-stimulated IL-17 production by CD4+ T cells. However, interleukin (IL) 17 production was induced by burn patient sera when it was previously treated with anti-IL-10 antibody. These results suggest that the high susceptibility to $C$. albicans infection observed in severe burn patients may be explained by the high levels of IL-10. However, any therapeutic intervention that aims to modulate IL-10 should be performed with caution in order to maintain the balance between inflammatory and anti-inflammatory molecules. Moreover, measuring IL10 and IL17 could be a useful strategy to determine which patients are more at risk of fungal infection. 


\section{Inhalation injury}

The pathophysiology of inhalation injury has also aroused interest. Recent work has assessed whether the severity of inhalation injury evokes an immune response measurable at a systemic level ${ }^{26}$. The results showed that plasma inflammatory markers were increased with worse inhalation injury severity, and that IL-1Ra had the strongest correlation with injury severity and outcomes. Other issues that have been examined are the factors that modify cell responses to soot, which are probably responsible for the development and severity of acute respiratory distress syndrome and lower respiratory tract infections. In this regard, it has been shown that high levels of IL-10 and IL-12p70 measured early in bronchoalveolar lavage fluid (BALF) of burn patients with inhalation injury may be associated with progression to more severe lung injury ${ }^{27}$. Moreover, these authors proposed a new hypothesis regarding inhalation injury pathophysiology: as Gram-negative pathogen infection was associated with more severe hypoxemia, it is likely that a prolonged increase in IL-10 levels of BAL fluid in these patients would increase immunosuppression and subsequent Gram-negative infection. On the other hand, cigarette smoking, which presents certain similarities to inhalation injury, may primarily trigger IL-33/ST2 production, which then stimulates the synthesis of further key pro-inflammatory cytokines, chemokines and mediators in the airway $^{28}$.

It has also been observed that bronchoalveolar lavage in burn patients with inhalation injury may present different inflammatory patterns according to the severity of the inhalation injury ${ }^{29}$. More importantly, pulmonary hyporesponsiveness has been associated with higher mortality after burn and inhalation injury ${ }^{30}$, suggesting that immunoparalysis is also important in the pathogenesis of inhalation injury and may serve as a potential therapeutic target ${ }^{31}$. 


\section{Wound healing}

Measuring biomarkers in burns may not only help to understand the pathophysiology of organ dysfunction development and inhalation injury, but may also indicate how the wound healing process occurs. Indeed, burn wound progression remains an important challenge in the overall management of these patients ${ }^{32}$. Recent experimental results suggested that the delayed wound healing observed in old animals is associated with an increase in keratinocyte 6 , matrix metalloproteinase-9 and CD44 $4^{33}$. Hence, it is likely that the modulation of these biomarkers could improve and accelerate wound healing in elderly patients. Other studies have examined the role of cell death in wound progression. Tan et al. ${ }^{34}$ demonstrated that autophagy and apoptosis are important features in wound progression at different time points. As autophagy precedes apoptosis, different treatment strategies should be applied at different time points during the burn injury depending on the primary mechanism involved in wound progression. Autophagic cell death has also been shown to occur early after severe burn ${ }^{35}$ and may play an important role in post-burn cardiac dysfunction. Interestingly, pharmacological inhibition of angiotensin II and ROS decreases autophagy and improves cardiac function ${ }^{35}$.

Interleukin 33 (IL33) has also been described as an important pathogenic feature in wound healing. Exogenous administration of IL33 accelerated wound healing and promoted collagen deposition and the expression of extracellular matrix (ECM)associated genes ${ }^{36}$, and also facilitated the development of activated macrophages in incisional wound tissue. Equally, administration of IL33 strongly inhibited methicillinresistant S. aureus (MRSA) colonization and accelerated cutaneous wound repair by promoting neutrophil proliferation and by enhancing collagen deposition and the expression of ECM-associated genes ${ }^{37}$. Thus, these results suggest that IL33 may have some effect on matrix synthesis and re-epithelialization during the wound repair 
process. All these results indicate that IL33 plays a key role in the wound healing process and could be considered as a potential therapeutic target.

Proinflammatory and profibrogenic cytokines are known to be active in hypertrophic scar development. In this regard, the expression of IL $1 \beta$ and type I receptors for TNF $\alpha$ in normal skin and post-burn normotrophic and hypertrophic scars has recently been assessed $^{38}$. The results showed that both IL-1 $\beta$ and type I receptor for TNFa were overexpressed in hypertrophic scars at the post-transcriptional level - the former associated primarily with keratinocytes and CD1a+ cells, and the latter in the blood vessels of hypertrophic scars.

\section{DIAGNOSIS OF COMPLICATIONS AND ASSESSMENT OF THERAPEUTIC INTERVENTIONS}

Fluid resuscitation

Appropriate fluid management is one of the most important issues in the immediate management of burn patients ${ }^{39}$. The aim of this fluid resuscitation is to correct the hypovolemic shock associated with the burn injury. However, avoiding overresuscitation is just as important as correcting the shock. Another study showed that fluid resuscitation of burn patients guided by intrathoracic blood volume index was associated with higher levels of IL6, IL6/IL10 ratio and the IL8/IL10 compared with urine output-guided resuscitation ${ }^{40}$. These results suggest that ITBVI-guided resuscitation may suppress the shift toward anti-inflammatory imbalance. Furthermore, several leukocyte surface markers were significantly higher expressed in the group guided by urine output. These results should make us become aware on the fact that therapeutic interventions may also affect biological processes and may also explain why some treatments succeed or fail in improving outcomes. 
Sepsis

Septic complications have been considered an important cause of death in burn patients ${ }^{41,42}$ and, therefore, their early identification may lead to early initiation of treatment and better outcomes. However, diagnosing sepsis in burn patients is still a challenge. In this scenario, several studies have examined the clinical utility of certain biomarkers for diagnosing sepsis and managing critically ill patients. The results of a recent study including more than 400 pediatric burn patients showed that interleukin-8 plasma concentration strongly correlates with the incidence of sepsis ${ }^{43}$. Moreover, patients with higher levels of IL8 had greater burn size, higher incidence of inhalation injury and multiorgan failure, and higher mortality.

On the other hand, a recent meta-analysis on the role of procalcitonin (PCT) in the diagnosis of sepsis and the prognosis of burn patients ${ }^{44}$ concluded that adding the determination of PCT to the diagnosis of sepsis may be beneficial. More recently, a large prospective cohort study of 145 burn patients aiming to examine the accuracy of PCT for diagnosing sepsis and localized infections ${ }^{45}$ found that maximum PCT plasma level was independently associated with mortality and that patients with septic shock presented higher concentrations of PCT at day 1. More interestingly, PCT levels were higher in patients with sepsis, respiratory tract infection and wound infection than prior to infection. Finally, PCT levels remained higher in septic burn patients with treatment failure, whereas successfully treated patients presented a substantial decrease in PCT concentration between the first and the third day of evolution.

In contrast, a recent study ${ }^{46}$ did not find PCT to be a useful marker of sepsis. Indeed, their results showed that $\mathrm{N}$-terminal pro-B-type natriuretic peptide significantly outperformed PCT in the diagnosis of sepsis. Moreover, stroke volume index and systemic vascular resistance index showed an excellent discriminative power for 
sepsis appearance in critically ill burn patients with TBSA equal to or greater than $15 \%$ who were intubated and had no previous cardiovascular comorbidities.

Another point of special interest is the ease of predicting and anticipating the occurrence of infection and sepsis in severe burns. Schultz et al. ${ }^{47}$ retrospectively analyzed a cohort of more than 100 burn patients comparing clinical variables that were associated with infection and sepsis. Using a classification and regression tree analysis, the results showed that heart rate $\geq 110 \mathrm{bpm}$, systolic blood pressure $\leq 100$ $\mathrm{mmHg}$ and intubation were the best predictors of sepsis. In contrast, a fraction of inhaled oxygen $>0.25$ and maximum temperature $\geq 39^{\circ} \mathrm{C}$ were the best predictors of infection.

\section{Inhalation}

In an experimental ovine model of burn injury and smoke inhalation, tocopherol nebulization improved oxygenation, pulmonary shunt fraction, respiratory mechanics, edema formation and bronchiolar obstruction ${ }^{48}$. This study also showed that tocopherol decreased the expression of a nitric oxide synthase inhibitor as well as arginase activity and collagen deposition. This effect helps to improve diffusion capacity and decreases edema formation. The authors conclude that inhalation injury was also mediated by reactive oxygen species and the arginase pathway, and that ROS scavengers such as tocopherol are potentially useful for treating these patients ${ }^{49}$.

More recently, serum concentrations of IL-6, IL-8 and TNF- $\alpha$ have been measured in patients with minor burns and smoke inhalation to assess whether high-frequency percussive ventilation (HFPV) increases inflammatory markers ${ }^{50}$. Interestingly, no increase in serum biomarkers was observed, suggesting that HFPV may not be harmful and could be considered as potential therapeutic tool for these patients. 
However, two recent randomized controlled trials of high-frequency oscillatory ventilation (HFOV) in ARDS patients did not find any benefit in mortality ${ }^{51,52}$.

\section{Abdominal compartment syndrome and renal failure}

Burn is an independent risk factor for intra-abdominal hypertension (IAH) and abdominal compartment syndrome (ACS) and the appearance of these conditions is associated with development of organ failure ${ }^{53}$ and higher mortality rates ${ }^{54}$. Moreover, as decreasing resuscitation volumes may reduce the prevalence of IAH and ACS, intra-abdominal pressure can be considered as another useful biomarker to guide fluid resuscitation in severe burn patients.

A biomarker may be especially useful if it can predict the development of organ failure before the clinical alteration appears. Early prediction of organ failure development may allow initiation of aggressive treatment that may limit its consequences. In this regard, acute renal failure (ARF) is a well-known complication of severe burns, especially ones with $\mathrm{IAH}$ and $\mathrm{ACS}^{53}$, and is an important cause of increased mortality ${ }^{55}$. Serum cystatin $\mathrm{C}$ and plasma and urine neutrophil gelatinase-associated lipocalin (NGAL) are known to rise 24 to 48 hours before the serum creatinine levels increase. However, in burns, only NGAL has been proven to be associated with development of early acute kidney injury and mortality ${ }^{56}$.

\section{DESIGNING NEW THERAPEUTIC STRATEGIES AND THE USE OF NEW THERAPEUTIC TARGETS}

As oxidative stress and neutrophil activation play important roles in the development and progression of burn injury, they can be considered as good therapeutic targets. Recently, a small randomized controlled trial analyzed the effect of $\mathrm{N}$-acetylcysteine in 
30 burn patients with more than $20 \%$ of total body surface area ${ }^{57}$. Although the study lacked sufficient power to draw conclusions regarding clinical outcomes, patients treated with $\mathrm{N}$-acetylcysteine presented lower levels of oxidant stress and plasma cytokines, and the treatment was associated with lower vasopressor requirement.

Recently, Stanojcic et al. ${ }^{58}$ investigated the effect of the nucleotide-binding domain, leucine-rich family (NLR), pyrin-containing 3 (NLRP3) inflammasome on insulin resistance and metabolic dysfunction in burn patients. Their results showed that the subcutaneous fat tissue of burn patients presented leucocyte infiltration, which was formed at least in part by monocytes. These monocytes presented increased inflammasome activity that was associated with higher levels of IL-1 $\beta$. These results provide new evidence regarding the role of the inflammasome in the genesis of stressinduced diabetes and the hypermetabolism related with severe burns, and identify it as a new therapeutic target.

On the other hand, it has been shown that burn injury directly decreases levels of tight junction proteins in the intestinal epithelium, leading to gut injury. This allows the movement of intraluminal contents across the mucosa, which can lead to the development of distant organ injury and multiple organ failure. In fact, the production of gut-derived proinflammatory mediators has been implicated in the shock and multiple organ failure associated with burn injury ${ }^{59}$. Interestingly, in rats with hemorrhagic shock, gut-derived factors that lead to organ injury and increased endothelial permeability were present at higher levels in mesenteric lymph than in the portal vein ${ }^{60}$. Moreover, the results of an experimental study showed that phosphodiesterase inhibition with pentoxifylline may attenuate burn-induced gut injury and, consequently, may protect against the organ failure associated with burn injury ${ }^{61}$. In contrast, ethanol intoxication at the time of burn injury suppresses intestinal immune defenses, impairs gut barrier functions and increases bacterial growth, and thus contributes significantly to the pathogenesis of post-burn distal organ injury ${ }^{59}$. These results suggest that levels of 
ethanol should be routinely measured in all burn patients at the moment of admission. In addition, recent experimental data has associated the expression of IL22 and other antimicrobial peptides with an increase in intestinal permeability ${ }^{62}$. Moreover, treatment with IL22 increase the expression of antimicrobial peptides, attenuates the increase in intestinal permeability and reduces bacterial load. These results suggest that IL22 contributes to gut epithelial and immune barrier functions following acute alcohol exposure and burn injury, and that the IL22/antimicrobial peptide pathway may be a useful therapeutic target in order to prevent sepsis and organ dysfunction in patients who present burn injury under alcohol influence.

\section{PROGNOSTIC VALUE}

Proteomic techniques facilitate the identification of protein profiles that can serve as diagnostic biomarkers or expression fingerprints for early assessment of predicted outcome as a result of disease or injury. This technique could be especially useful in diseases that involve multiple organs, such as burn injury or critical illness. Finnerty et $\mathrm{al}^{63}$ determined the proteomic survival signature following severe burn injury using high-resolution liquid chromatography-mass spectrometry (LC-MS) and multiplex cytokine analysis. They observed that 43 proteins were significantly altered in nonsurvivors. These proteins were involved in various biological pathways such as the coagulation cascade, complement response, hepatic acute-phase response signaling and inflammation. Interestingly, the majority of these proteins have not been previously associated with burn injury, suggesting that LC-MS is useful for describing proteome signatures and for identifying new mechanisms related with survival after burn injury.

More recently, a large study including severely burned children with more than $30 \%$ of total body surface affected aimed to determine whether a panel of common biomarkers 
could be used to predict survival ${ }^{18}$. Non-survivors had significantly higher levels of biomarkers such as IL-6, IL-8, granulocyte colony-stimulating factor, monocyte chemoattractant protein-1, C-reactive protein, glucose, insulin, blood urea nitrogen, creatinine, and bilirubin. Therefore, these biomarkers can be used for monitoring patients and for identifying those at high risk who will benefit from more aggressive treatment. Furthermore, non-survivors are more likely to present sepsis and multiorgan failure, suggesting that these biomarkers could be involved in the pathogenesis of these conditions. Previously, it was shown that the inflammatory and metabolic response is dependent on burn size ${ }^{64}$; the greater the extent of surface area affected, the higher the inflammation and metabolic response. In addition, patients with a greater percentage of surface burned presented higher indices of sepsis and inhalation injury, higher mechanical ventilation requirement, and increased incidence of myocardial depression and mortality. These authors suggested that patients with a greater extent of body surface burned presented higher levels of proinflammatory cell mediators that trigger and enhance the hypermetabolic response, thus leading to a worse outcome. Hypoglycemia has also recently been described as a good prognostic marker ${ }^{65}$. One hundred and sixty-six pediatric burn patients who presented one or more episode of hypoglycemia during the first 60 days of admission were matched with patients without hypoglycemia using a propensity score. Matched patients who presented one or more episode of hypoglycemia had greater inflammatory and metabolic response and higher prevalence of sepsis, multiorgan failure and death. Hence, the implementation of therapeutic strategies that minimize the appearance of this complication is imperative. Hyperlactatemia has been considered as a marker of tissue hypoperfusion. Moreover, the Sepsis Surviving Campaign ${ }^{66}$ proposed targeting fluid resuscitation to normalize lactate levels in those patients with initial hyperlactatemia. In burn patients, baseline lactate levels have been reported to predict mortality and their normalization during the first 24 hours of admission is associated with better survival ${ }^{67}$. 
More recently, a model combining clinical characteristics and a panel of genomic biomarkers identified burn patients who were at high risk of developing repeated infections $^{68}$. The genomic signature provides new information about susceptibility to infection and, therefore, may lead to the description of new therapeutic targets. 


\section{FUTURE DIRECTIONS}

Research in biomarkers is continuously evolving. The introduction of techniques such as metabolomics and proteomics may make it possible to detect new biomarkers that are even more sensitive and specific than the ones we have at present. Moreover, the genomic fingerprint may also have an important role to play.

The first aim of research into new biomarkers should be to prevent organ dysfunction, which is one of the key determinants of morbidity and mortality in burn patients. Thus, research in new biomarkers that can predict the appearance of MODS before it occurs is imperative. Second, biomarkers may be useful for stratifying patients according to severity. Correct stratification of patients in terms of severity is the only way to ensure that the right treatment is delivered to the right patient, and will allow us to select high risk patients who are more likely to benefit from more aggressive treatments. Third, it is important to validate new experimental findings clinically, which, in fact, is the key point of translational research. In contrast, experimental research may help to demonstrate the hypothesis generated in clinical studies, as it was the case with the hypothesis that an injurious mechanical ventilation may cause distal organ dysfunction ${ }^{69}$. Thus, experimental research can be helpful in describing the exact mechanisms involved in organ dysfunction generation after burn injury. And finally, the description of new biomarkers will help to develop new therapeutic targets. In this regard, as in the case of the acute respiratory distress syndrome ${ }^{70}$, the use of modified stem cells overexpressing proteins that could enhance wound healing by blocking certain inflammatory pathways may be a point of special interest. 


\section{CONCLUSIONS}

Several inflammatory mediators have been shown to be increased after burn injury. They may be important in burn pathophysiology, contributing to organ dysfunction and sepsis apparition, and they may also predict outcomes. Moreover, they have been involved in pathophysiology of some special processes, such as inhalation injury or wound healing. Consequently, some biomarkers have been described as potential therapeutic targets. Importantly, as therapeutic interventions may also affect biological processes, biomarkers may be a useful tool to guide some treatments and may also explain why some treatments succeed or fail in improving outcomes. Therefore, investigation into biomarkers in severe burn patients is a key feature of translational medicine in this area of knowledge. Future research should focus preventing complications of burn injury, stratifying patients according to their risk and severity, designing new therapeutic targets and validating clinically new experimental findings. 


\section{ACKNOWLEDGMENTS}

This work was supported by grants from Instituto de Salud Carlos III-FEDER, (PI14/01420). 


\section{References}

1. Brusselaers N, Monstrey S, Vogelaers D, Hoste E, Blot S. Severe burn injury in Europe: a systematic review of the incidence, etiology, morbidity, and mortality. Crit. Care 2010;14(5):R188. doi:10.1186/cc9300.

2. Mock C, Peck M, Peden M, Krug E, eds. A WHO plan for burn prevention and care. Geneva, World Health Organization, 2008.

3. Wasiak J, Lee SJ, Paul E, et al. Predictors of health status and health-related quality of life 12 months after severe burn. Burns 2014;40(4):568-74. doi:10.1016/j.burns.2014.01.021.

4. Puntmann VO. How-to guide on biomarkers: biomarker definitions, validation and applications with examples from cardiovascular disease. Postgrad. Med. J. 2009;85(1008):538-45. doi:10.1136/pgmj.2008.073759.

5. WHO International Programme on Chemical Safety Biomarkers and Risk Assessment: Concepts and Principles. 1993. Retrieved from http://www.inchem.org/documents/ehc/ehc/ehc155.htm.

6. Mandrup-Poulsen T, Wogensen LD, Jensen M, et al. Circulating interleukin-1 receptor antagonist concentrations are increased in adult patients with thermal injury. Crit. Care Med. 1995;23(1):26-33. Available at:

http://www.ncbi.nlm.nih.gov/pubmed/8001382. Accessed August 3, 2015.

7. Endo S, Inada K, Yamada Y, et al. Plasma levels of interleukin-1 receptor antagonist (IL-1ra) and severity of illness in patients with burns. J. Med. 1996;27(1-2):57-71. Available at: http://www.ncbi.nlm.nih.gov/pubmed/8863178. Accessed August 3, 2015.

8. Vindenes HA, Ulvestad E, Bjerknes R. Concentrations of cytokines in plasma of patients with large burns: their relation to time after injury, burn size, inflammatory variables, infection, and outcome. Eur. J. Surg. 1998;164(9):64756. doi:10.1080/110241598750005525.

9. Yeh FL, Lin WL, Shen HD, Fang RH. Changes in serum tumour necrosis factoralpha in burned patients. Burns 1997;23(1):6-10. Available at:

http://www.ncbi.nlm.nih.gov/pubmed/9115613. Accessed August 3, 2015.

10. Yeh FL, Lin WL, Shen HD, Fang RH. Changes in levels of serum IL-8 in burned patients. Burns 23(7-8):555-9. Available at:

http://www.ncbi.nlm.nih.gov/pubmed/9568323. Accessed August 3, 2015.

11. Yeh FL, Lin WL, Shen HD, Fang RH. Changes in circulating levels of interleukin 6 in burned patients. Burns 1999;25(2):131-6. Available at: http://www.ncbi.nlm.nih.gov/pubmed/10208387. Accessed August 3, 2015.

12. Yeh FL, Lin WL, Shen HD. Changes in circulating levels of an anti-inflammatory cytokine interleukin 10 in burned patients. Burns 2000;26(5):454-9. Available at: http://www.ncbi.nlm.nih.gov/pubmed/10812267. Accessed August 3, 2015. 
13. Yeh FL, Shen HD, Fang RH. Deficient transforming growth factor beta and interleukin-10 responses contribute to the septic death of burned patients. Burns 2002;28(7):631-7. Available at: http://www.ncbi.nlm.nih.gov/pubmed/12417156. Accessed August 3, 2015.

14. Jeschke MG, Chinkes DL, Finnerty CC, et al. Pathophysiologic response to severe burn injury. Ann. Surg. 2008;248(3):387-401. doi:10.1097/SLA.0b013e3181856241.

15. Hettiaratchy S, Dziewulski P. ABC of burns: pathophysiology and types of burns. BMJ 2004;328(7453):1427-1429.

16. Flierl M a, Stahel PF, Touban BM, et al. Bench-to-bedside review: Burn-induced cerebral inflammation--a neglected entity? Crit. Care 2009;13(3):215. doi:10.1186/cc7794.

17. Kraft R, Herndon DN, Finnerty CC, Shahrokhi S, Jeschke MG. Occurrence of multiorgan dysfunction in pediatric burn patients: incidence and clinical outcome. Ann. Surg. 2014;259(2):381-7. doi:10.1097/SLA.0b013e31828c4d04.

18. Jeschke MG, Gauglitz GG, Finnerty CC, Kraft R, Mlcak RP, Herndon DN. Survivors versus nonsurvivors postburn: differences in inflammatory and hypermetabolic trajectories. Ann. Surg. 2014;259(4):814-23. doi:10.1097/SLA.0b013e31828dfbf1.

19. Bloemsma GC, Dokter J, Boxma H, Oen IMMH. Mortality and causes of death in a burn centre. Burns 2008;34(8):1103-7. doi:10.1016/j.burns.2008.02.010.

20. Saffle JR, Sullivan JJ, Tuohig GM, Larson CM. Multiple organ failure in patients with thermal injury. Crit. Care Med. 1993;21(11):1673-83. Available at: http://www.ncbi.nlm.nih.gov/pubmed/8222683. Accessed October 19, 2014.

21. Csontos C, Foldi V, Pálinkas L, et al. Time course of pro- and anti-inflammatory cytokine levels in patients with burns--prognostic value of interleukin-10. Burns 2010;36(4):483-94. doi:10.1016/j.burns.2009.10.009.

22. Szelig L, Rendeki S, Foldi V, Lantos J, Bogar L, Csontos C. Time course of CD marker expression in patients with burns and its prognostic value. Burns 2014;40(4):575-82. doi:10.1016/j.burns.2013.09.023.

23. Mason AD, McManus AT, Pruitt BA. Association of burn mortality and bacteremia. A 25-year review. Arch. Surg. 1986;121(9):1027-31. Available at: http://www.ncbi.nlm.nih.gov/pubmed/3741098. Accessed September 7, 2014.

24. Horvath EE, Murray CK, Vaughan GM, et al. Fungal wound infection (not colonization) is independently associated with mortality in burn patients. Ann. Surg. 2007;245(6):978-85. doi:10.1097/01.sla.0000256914.16754.80.

25. Inatsu A, Kogiso M, Jeschke MG, et al. Lack of Th17 cell generation in patients with severe burn injuries. J. Immunol. 2011;187(5):2155-61. doi:10.4049/jimmunol.1003235. 
26. Davis CS, Janus SE, Mosier MJ, et al. Inhalation injury severity and systemic immune perturbations in burned adults. Ann. Surg. 2013;257(6):1137-46. doi:10.1097/SLA.0b013e318275f424.

27. Jones SW, Zhou H, Ortiz-Pujols SM, et al. Bronchoscopy-derived correlates of lung injury following inhalational injuries: a prospective observational study. PLoS One 2013;8(5):e64250. doi:10.1371/journal.pone.0064250.

28. Qiu C, Li Y, Li M, et al. Anti-interleukin-33 inhibits cigarette smoke-induced lung inflammation in mice. Immunology 2013;138(1):76-82. doi:10.1111/imm.12020.

29. Albright JM, Davis CS, Bird MD, et al. The acute pulmonary inflammatory response to the graded severity of smoke inhalation injury. Crit. Care Med. 2012;40(4):1113-21. doi:10.1097/CCM.0b013e3182374a67.

30. Davis CS, Albright JM, Carter SR, et al. Early pulmonary immune hyporesponsiveness is associated with mortality after burn and smoke inhalation injury. J. Burn Care Res. 33(1):26-35. doi:10.1097/BCR.0b013e318234d903.

31. Leentjens J, Kox M, Koch RM, et al. Reversal of immunoparalysis in humans in vivo: a double-blind, placebo-controlled, randomized pilot study. Am. J. Respir. Crit. Care Med. 2012;186(9):838-45. doi:10.1164/rccm.201204-0645OC.

32. Shupp JW, Nasabzadeh TJ, Rosenthal DS, Jordan MH, Fidler P, Jeng JC. A review of the local pathophysiologic bases of burn wound progression. J. Burn Care Res. 31(6):849-73. Available at: http://www.ncbi.nlm.nih.gov/pubmed/21105319. Accessed September 3, 2014.

33. Simonetti O, Oriana S, Lucarini G, et al. Delayed wound healing in aged skin rat models after thermal injury is associated with an increased MMP-9, K6 and CD44 expression. Burns 2013;39(4):776-87. doi:10.1016/j.burns.2012.09.013.

34. Tan J-Q, Zhang H-H, Lei Z-J, et al. The roles of autophagy and apoptosis in burn wound progression in rats. Burns 2013;39(8):1551-6. doi:10.1016/j.burns.2013.04.018.

35. Xiao R, Teng M, Zhang Q, Shi X, Huang Y. Myocardial autophagy after severe burn in rats. PLoS One 2012;7(6):e39488. doi:10.1371/journal.pone.0039488.

36. Yin $\mathrm{H}, \mathrm{Li} X, \mathrm{Hu}$ S, et al. IL-33 accelerates cutaneous wound healing involved in upregulation of alternatively activated macrophages. Mol. Immunol. 2013;56(4):347-53. doi:10.1016/j.molimm.2013.05.225.

37. Yin H, Li X, Hu S, et al. IL-33 promotes Staphylococcus aureus-infected wound healing in mice. Int. Immunopharmacol. 2013;17(2):432-8. doi:10.1016/j.intimp.2013.07.008.

38. Salgado RM, Alcántara L, Mendoza-Rodríguez CA, et al. Post-burn hypertrophic scars are characterized by high levels of IL-1 $\beta$ mRNA and protein and TNF- $\alpha$ type I receptors. Burns 2012;38(5):668-76. doi:10.1016/j.burns.2011.12.012. 
39. Snell JA, Loh N-HW, Mahambrey T, Shokrollahi K. Clinical review: the critical care management of the burn patient. Crit. Care 2013;17(5):241.

doi:10.1186/cc12706.

40. Foldi V, Lantos J, Bogar L, Roth E, Weber G, Csontos C. Effects of fluid resuscitation methods on the pro- and anti-inflammatory cytokines and expression of adhesion molecules after burn injury. J. Burn Care Res. 31(3):48091. doi:10.1097/BCR.0b013e3181db527a.

41. Sharma BR, Harish D, Singh VP, Bangar S. Septicemia as a cause of death in burns: an autopsy study. Burns 2006;32(5):545-9.

doi:10.1016/j.burns.2006.02.008.

42. Barrow RE, Przkora R, Hawkins HK, Barrow LN, Jeschke MG, Herndon DN. Mortality related to gender, age, sepsis, and ethnicity in severely burned children. Shock 2005;23(6):485-7. Available at:

http://www.ncbi.nlm.nih.gov/pubmed/15897798. Accessed March 14, 2015.

43. Kraft R, Herndon DN, Finnerty CC, Cox RA, Song J, Jeschke MG. Predictive Value of IL-8 for Sepsis and Severe Infections After Burn Injury - A Clinical Study. Shock 2014. doi:10.1097/SHK.0000000000000294.

44. Mann E a, Wood GL, Wade CE. Use of procalcitonin for the detection of sepsis in the critically ill burn patient: a systematic review of the literature. Burns 2011;37(4):549-58. doi:10.1016/j.burns.2010.04.013.

45. Lavrentieva a, Papadopoulou S, Kioumis J, Kaimakamis E, Bitzani M. PCT as a diagnostic and prognostic tool in burn patients. Whether time course has a role in monitoring sepsis treatment. Burns 2012;38(3):356-63.

doi:10.1016/j.burns.2011.08.021.

46. Paratz JD, Lipman J, Boots RJ, Muller MJ, Paterson DL. A New Marker of Sepsis Post Burn Injury?*. Crit. Care Med. 2014;42(9):2029-2036. doi:10.1097/CCM.0000000000000400.

47. Schultz L, Walker S a N, Elligsen M, et al. Identification of predictors of early infection in acute burn patients. Burns 2013;39(7):1355-66. doi:10.1016/j.burns.2013.04.009.

48. Yamamoto Y, Enkhbaatar P, Sousse LE, et al. Nebulization with y-tocopherol ameliorates acute lung injury after burn and smoke inhalation in the ovine model. Shock 2012;37(4):408-14. doi:10.1097/SHK.0b013e3182459482.

49. Yamamoto $Y$, Sousse LE, Enkhbaatar $P$, et al. $Y$-tocopherol nebulization decreases oxidative stress, arginase activity, and collagen deposition after burn and smoke inhalation in the ovine model. Shock 2012;38(6):671-6. doi:10.1097/SHK.0b013e3182758759.

50. Reper P, Heijmans W. High-frequency percussive ventilation and initial biomarker levels of lung injury in patients with minor burns after smoke inhalation injury. Burns 2014:2-7. doi:10.1016/j.burns.2014.05.007. 
51. Ferguson ND, Cook DJ, Guyatt GH, et al. High-frequency oscillation in early acute respiratory distress syndrome. N. Engl. J. Med. 2013;368(9):795-805. doi:10.1056/NEJMoa1215554.

52. Young D, Lamb SE, Shah S, et al. High-frequency oscillation for acute respiratory distress syndrome. N. Engl. J. Med. 2013;368(9):806-13. doi:10.1056/NEJMoa1215716.

53. Ruiz-Castilla M, Barret JP, Sanz D, et al. Analysis of intra-abdominal hypertension in severe burned patients: the Vall d'Hebron experience. Burns 2014;40(4):719-24. doi:10.1016/j.burns.2013.09.021.

54. Strang SG, Van Lieshout EMM, Breederveld RS, Van Waes OJF. A systematic review on intra-abdominal pressure in severely burned patients. Burns 2014;40(1):9-16. doi:10.1016/j.burns.2013.07.001.

55. Holm C, Hörbrand F, von Donnersmarck GH, Mühlbauer W. Acute renal failure in severely burned patients. Burns 1999;25(2):171-8. Available at: http://www.ncbi.nlm.nih.gov/pubmed/10208394. Accessed December 9, 2014.

56. Yang HT, Yim H, Cho YS, et al. Assessment of biochemical markers in the early post-burn period for predicting acute kidney injury and mortality in patients with major burn injury: comparison of serum creatinine, serum cystatin-C, plasma and urine neutrophil gelatinase-associated lipoca. Crit. Care 2014;18(4):R151. doi:10.1186/cc13989.

57. Csontos C, Rezman B, Foldi V, et al. Effect of N-acetylcysteine treatment on oxidative stress and inflammation after severe burn. Burns 2012;38(3):428-37. doi:10.1016/j.burns.2011.09.011.

58. Stanojcic $\mathrm{M}$, Chen $\mathrm{P}$, Harrison $\mathrm{R}$ a, et al. Leukocyte infiltration and activation of the NLRP3 inflammasome in white adipose tissue following thermal injury. Crit. Care Med. 2014;42(6):1357-64. doi:10.1097/CCM.0000000000000209.

59. Choudhry MA, Chaudry IH. Alcohol, burn injury, and the intestine. J. Emerg. Trauma. Shock 2008;1(2):81-7. doi:10.4103/0974-2700.43187.

60. Magnotti LJ, Upperman JS, Xu DZ, Lu Q, Deitch EA. Gut-derived mesenteric lymph but not portal blood increases endothelial cell permeability and promotes lung injury after hemorrhagic shock. Ann. Surg. 1998;228(4):518-27. Available at:

http://www.pubmedcentral.nih.gov/articlerender.fcgi?artid=1191527\&tool=pmcen trez\&rendertype=abstract. Accessed April 2, 2015.

61. Costantini TW, Loomis WH, Putnam JG, et al. Burn-induced gut barrier injury is attenuated by phosphodiesterase inhibition: effects on tight junction structural proteins. Shock 2009;31(4):416-22. doi:10.1097/SHK.0b013e3181863080.

62. Rendon JL, Li X, Akhtar S, Choudhry MA. Interleukin-22 modulates gut epithelial and immune barrier functions following acute alcohol exposure and burn injury. Shock 2013;39(1):11-8. doi:10.1097/SHK.0b013e3182749f96. 
63. Finnerty CC, Jeschke MG, Qian W-J, et al. Determination of burn patient outcome by large-scale quantitative discovery proteomics. Crit. Care Med. 2013;41(6):1421-34. doi:10.1097/CCM.0b013e31827c072e.

64. Jeschke MG, Mlcak RP, Finnerty CC, et al. Burn size determines the inflammatory and hypermetabolic response. Crit. Care 2007;11(4):R90. doi:10.1186/cc6102.

65. Jeschke MG, Pinto R, Herndon DN, Finnerty CC, Kraft R. Hypoglycemia is associated with increased postburn morbidity and mortality in pediatric patients. Crit. Care Med. 2014;42(5):1221-31. doi:10.1097/CCM.0000000000000138.

66. Dellinger RP, Levy MM, Rhodes A, et al. Surviving Sepsis Campaign: international guidelines for management of severe sepsis and septic shock, 2012. Intensive Care Med. 2013;39(2):165-228. doi:10.1007/s00134-012-27698.

67. Andel D, Kamolz L-P, Roka J, et al. Base deficit and lactate: early predictors of morbidity and mortality in patients with burns. Burns 2007;33(8):973-8. doi:10.1016/j.burns.2007.06.016.

68. Yan S, Tsurumi A, Que Y-A, et al. Prediction of Multiple Infections After Severe Burn Trauma: A Prospective Cohort Study. Ann. Surg. 2014;00(00). doi:10.1097/SLA.0000000000000759.

69. Imai Y, Parodo J, Kajikawa O, et al. Injurious mechanical ventilation and endorgan epithelial cell apoptosis and organ dysfunction in an experimental model of acute respiratory distress syndrome. JAMA 289(16):2104-12. doi:10.1001/jama.289.16.2104.

70. Martínez-González I, Roca O, Masclans JR, et al. Human mesenchymal stem cells overexpressing the IL-33 antagonist soluble IL-1 receptor-like-1 attenuate endotoxin-induced acute lung injury. Am. J. Respir. Cell Mol. Biol. 2013;49(4):552-62. doi:10.1165/rcmb.2012-0406OC. 